\title{
A Design to Filter Undesired Message from OSN User Wall
}

\author{
Ganesh Ghone ${ }^{1}$, Pratiksha Pawar ${ }^{2}$ \\ Professor, Computer Science \& Engg, DACOE, Karad, India ${ }^{1}$ \\ Student, Computer Science \& Engg, DACOE, Karad, India ${ }^{2}$
}

\begin{abstract}
One of the important issue in today's world is nothing but users ability to take the limitations posted on the on their private wall. Now a day OSN provides little support to OSN posting. Proposed system able to direct control on the user's vulgar messages, for this system we use filtering mechanism are their walls to delete the existing system problem. Machine method used to direct control message posted on the wall. For this phenomenon we use machine learning based soft classifier that that work with the help of the content based filtering algorithm. OSN is mostly popular younger generation entertainment .It helps of content based filtering algorithm. It helps to communicate the peoples, friends as well as society through online order. The proposed system helps to keep the track of the message for posting on the wall of online social network. On the point of exiting system and need of today's life, my paper work for filter unwanted message before they rich user wall.
\end{abstract}

Keywords: Social Network Application, SNM- Social Network Manager, black List, FL- filter Wall.

\section{INTRODUCTION}

Most important and attractive medium to communicate with each other is information or number of content will be audio, video, image, pdf etc. As compare to until 1 number days world. Content will be very vast filtering of information is used OSN provide very little support to filter unwanted message from OSN user walls for unrelated purpose[1].

This gives the ability of user to automatically control on the user walls. We use machine learning technique to filter or categorized message on public wall. Short text classifier is related with the process of extraction and selection of set o characterization. Also system contains the support of blacklist and filtering wall[3].

On extra example face book allows all users to posting any vulgar message on the public or private wall. However no content based preference is supported and therefore smaller support to prevent message on the public and private areas.

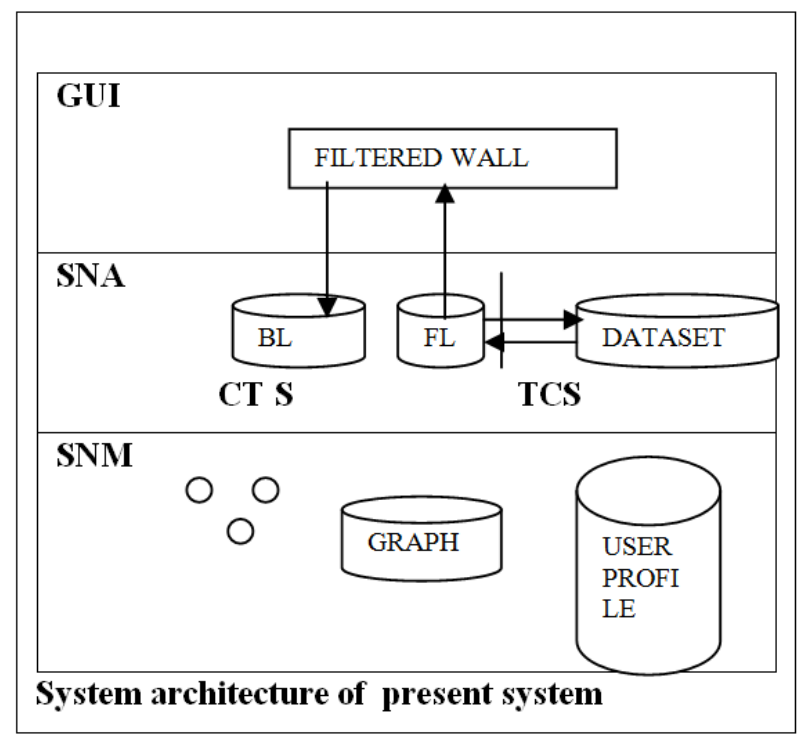

In OSNs, there are some off the peoples will be present on OSNs, that will connected to each other. In that they are commenting and posting other posts on particular public or private users wall that area or space is called as general walls [3].

GUI - graphical user interface.

SNA - Social Network Application.

SNM- Social Network Manager.

BL- black List.

FL- filter Wall.

CTS- content text classifier.

According to face book, there are different types of information or data (such as news, web contents, texts or web links) transferred to the users private own space. That posts may be vulgar, offensive, violence, unrated or undesired etc. The filtered message will be displayed on the user's private or public areas this place or space is also called as filtered wall (FW).

In this filtering rules, which is users can being what contents should not be displayed on their walls[4]. In which, we can create blacklist, that will perform as a filtering the some contents of the messages and we can check either message should be unrated or not. If this message content will be unrated then message will be not displayed to user's wall or filtered wall. If else this 
IARJSET

message contents will be not unrated means it is simple Filtering Process:

message, that is the Hii, then this message will be In filtering process some words are not correct words for displayed users wall or filtered wall[3].

\section{BACKGROUND OF MOTIVATION}

The main important concept for motivation is data mining. Data mining is the concept that choose or selecting the knowledgeable information for the large amount of data. In the proposed system large statement have vulgar word, by using data mining technique we select the vulgar word block whole message for prevent the message on wall

\section{RELATED WORK AND LITERATURE VIEW}

In this technique filtering technology means any user can filter single word, statement or paragraph. Can be check or filter. Information filtering is based on the two criteria: content-based \& information -based filtering from this filtering user can satisfied for their evolution[1]. It can provide easy \&comfortable work assessment to the user. This is based on machine learning technique. In contentbased filtering, there will be checking of individual words. In information filtering there is checking of complete sentence [3].

\section{EXISTING SYSTEM}

In existing system there is very little support to prevent the undesired message from user's walls[4]. There is no content based filtering as well as black list concept to prevent the message on their public or private walls.

\section{Drawback:}

1. There are do not have any restriction on their post and comment.

2. It requires Adhoc classification strategy for categorization technique.

3. It content vulgar word on the commuting the public wall.

\section{PROPOSED WORK}

1) System able to work on vulgar words

2 ) Automatic control the messages on users. It private and public wall.

3) It uses black list and messages creator relationship and characteristics.

4 )It help FW rules specifications in the classification of process.

\section{METHODOLOGY}

\section{Blacklist:}

Blacklist is managed by system for avoiding the bad words on the user's timeline. The bad words are stored in the database. Words are checked by blacklist, if word is present in blacklist then that word is block. user's that are checked in filtering process. [4] This process is used for avoiding the bad words on another user's timeline.

Screen will be generated are as like follow.
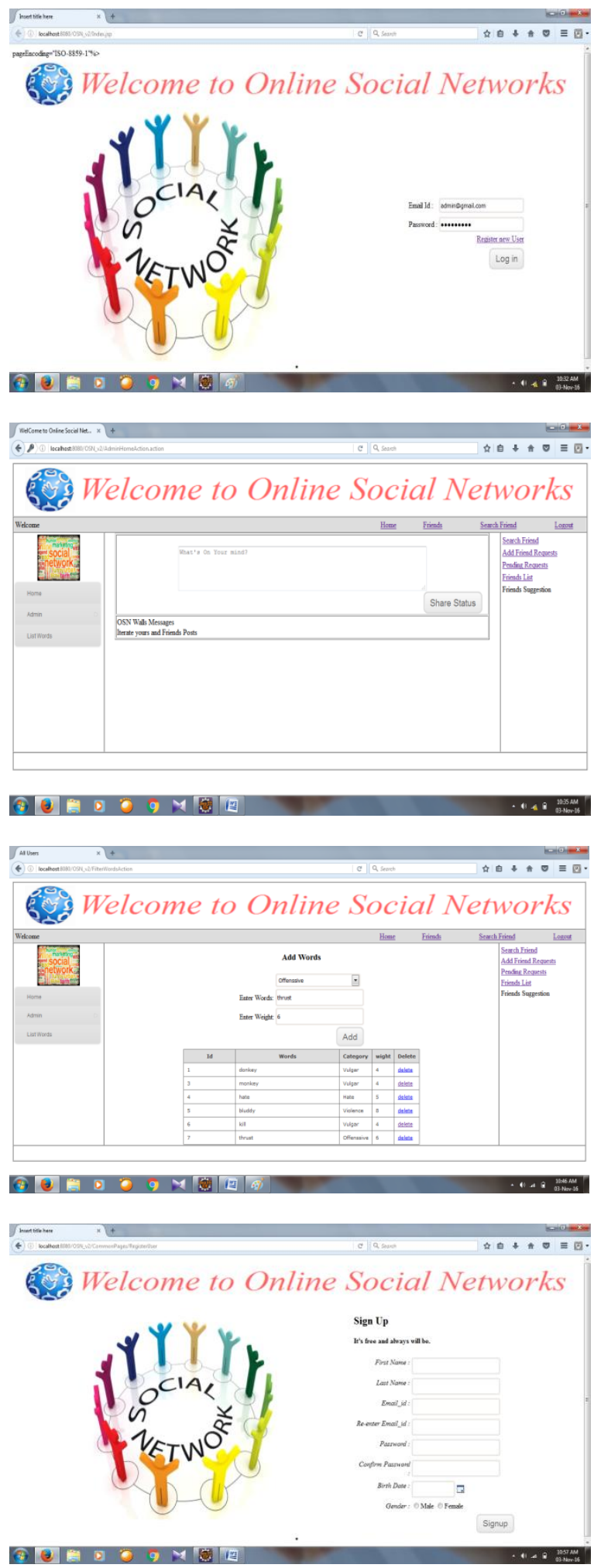
IARJSET

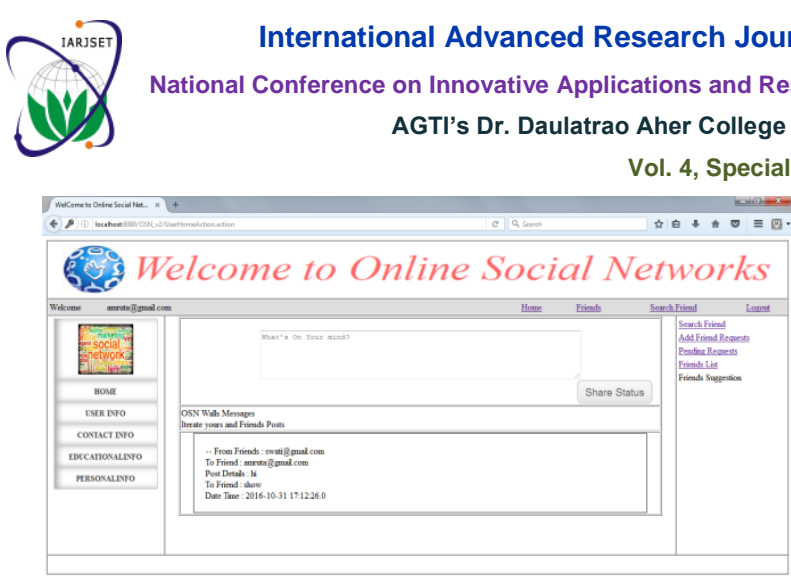

9)

\section{CONCLUSION}

As compare to the existing system, our proposed system work properly. Here are some incrementing progresses in the filtering and blacklist process we are using content bases filtering which supports for avoidance of bad words posting on user's timeline. When user can post a message on another user's wall then our system check that message $\&$ if there is occurrence of bad word then this message will be blocked i.e. cannot be displayed further.

\section{REFERENCES:}

[1] Marco Vanetti, Elisabetta Binaghi, Elena Ferrari, Barbara Carminati, an Moreno Carullo, "A System to Filter Unwanted Messages from OSN User Walls", 2013

[2] F. Sebastiani, -"Machine learning in automated text categorization" ACM Computing Surveys, vol.34, no. 1, pp. 1-47, 2002.

[3] R.J. Mooney and L. Roy, "Content-Based Book Recommending Using Learning for Text Categorization," Proc. Fifth ACM Conf. Digital Libraries, pp. 195-204, 2000.

[4] F. Sebastiani, "Machine Learning in Automated Text Categorization," ACM Computing Surveys, vol. 34, no. 1, pp. 1-47, 2002.

[5] M. Chau and H. Chen, "A Machine Learning Approach to Web Page Filtering Using Content and Structure Analysis," Decision Support Systems, vol. 44, no. 2, pp. 482-494, 2008.

[6] S. Pollock, "A Rule-Based Message Filtering System," ACM Trans.Office Information Systems, vol. 6, no. 3, pp. 232-254, 1988.

\section{BIOGRAPHIES}

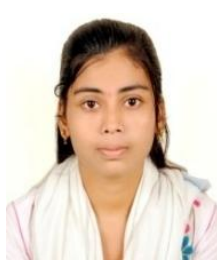

Pratiksha Pawar is a BE computer science\&Engineering student of G.K. Gujar Memorial Charitable Trust's DR.Daulatrao Aher College Of Engineering ,Karad, India in 2017 from Shivaji University, Kolhapur.

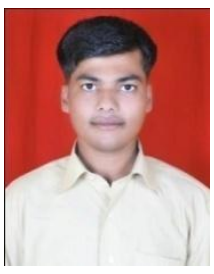

Ganesh Ghone is assistant professor in Computer Science \& Engineering department of G.K. Gujar Memorial Charitable Trust's DR. Daulatrao Aher College Of Engineering ,Karad, India. 